\title{
Un sistema de información geográfico para el transporte público basado en el estandar GTFS realtime
}

\author{
A geographic information system for public transportation \\ based on GTFS realtime standard
}

\author{
Andrés Colque $^{1} \quad$ Ricardo Valdivia $^{1 *} \quad$ Mónica Navarrete $^{2} \quad$ Sebastián Aracena $^{1}$
}

Recibido 29 de noviembre de 2018, aceptado 29 de noviembre de 2019

Received: November 29, 2018 Accepted: November 29, 2019

\section{RESUMEN}

A partir de la experiencia obtenida en el desarrollo del sistema Web SIG SITPA, un sistema planificador de viajes para el transporte público de la ciudad de Arica, basado en el estándar GTFS, se implementó un sistema que permite realizar un seguimiento, en tiempo real, de taxibuses mediante el uso de GPS.

El Sistema de Información Geográfico de Transporte (SIG-T), propuesto en este artículo, fue desarrollado a partir de la integración de software de libre disposición y se basa en el uso del estándar GTFS realtime. Su uso permite, no sólo la visualización de la posición actual de los taxibuses en el mapa de la ciudad, sino que también, permite predecir la llegada de estos a paraderos y la incorporación de mensajes de alertas asociados a problemas en la ruta.

Este sistema espera ser un aporte en el ámbito de los SIG-T, dado que es una solución de bajo costo que puede ser implementada en diferentes ciudades del país y cuya aplicación debería incentivar el uso del Transporte Público considerando, además, una potencial implementación de paraderos inteligentes.

Palabras clave: SIG-T, transporte público, software de libre disposición, AVL, GTFS realtime.

\begin{abstract}
Based on the experience in the development of the GIS SITPA Web system, a travel planner for the public transport in the city of Arica, based on the GTFS standard, a system was implemented that allows realtime tracking of taxi-buses by using GPS.

The Geographic Information System of Transport (SIG-T, for its acronym in Spanish), suggested in this article, was developed from the integration of freely available software and is based on the use of GTFS in real time. Its use allows, not only the visualization of the current position of the taxi-buses on the map of the city, but also, it allows to predict their arrival to the bus stops and to incorporate alert messages related with problems on the route.

This system aims to be a contribution in the scope of the SIG-T, since it is a low-cost solution that can be implemented in different cities of the country and its application can encourage the use of public transport and a potential implementation of smart bus stops.
\end{abstract}

Keywords: SIG-T, public transport, open source software, AVL, GTFS realtime.

1 Universidad de Tarapacá. Facultad de Ingeniería. Departamento de Ingeniería en Computación e Informática. Arica, Chile. E-mail: amos.colque@gmail.com; rvaldivi@uta.cl; sebastian.andres.aracena@gmail.com

2 Universidad de Tarapacá. Facultad de Administración y Economía. Escuela de Administración y Negocios. Arica, Chile. E-mail: mnavarre@uta.cl

* Autor de correspondencia: rvaldivi@uta.cl 


\section{INTRODUCCIÓN}

Actualmente en la ciudad de Arica - Chile existen aplicaciones de tipo Web que entregan información del servicio del transporte público, no obstante, estas no permiten predecir, en tiempo real, la llegada de cada taxibus a un paradero en particular. $\mathrm{La}$ herramienta descrita en este artículo se orienta a entregar este tipo de información, permitiendo la potencial implementación de paraderos inteligentes.

Las ciudades modernas aspiran a ser ciudades inteligentes [1] que incorporen, entre otras facilidades, elementos que faciliten a los usuarios, el desplazamiento por ella de forma fácil e independiente.

GTFS (General Transist Feed Specification) es un estándar que define un formato común para los horarios de transporte público y la información geográfica asociada a ellos. El uso de GTFS permite que las empresas de transporte público publiquen sus datos de transporte y que los programadores escriban aplicaciones que los consuman [2]. Esta propuesta considera, adicionalmente, el uso de la especificación GTFS realtime. Esta permite que los sistemas incorporen información asociada a las posiciones de vehículos, actualizaciones de viaje y alertas de servicio. Para esto, se requiere del uso de un sistema AVL (Automatic Vehicle Location) del transporte de taxibuses, el que captura datos de posición vehicular a través de dispositivos GPS.

Este articulo describe cómo implementar un Sistema Información Geográfica para el Transporte (SIG-T) [3] basado en el uso del estándar GTFS realtime, detallando los distintos procesos y herramientas (en su mayoría open source) que se utilizaron en su desarrollo. El sistema Web frond-end desarrollado integra varios sistemas que permiten visualizar los datos de GTFS realtime en interfaces útiles para el usuario. En particular, las interfaces de predicciones de llegadas en los paraderos, la interfaz de seguimiento de los taxibuses y las interfaces de las alertas que están asociadas a una ruta. Este sistema se ha desarrollado en PHP usando el framework Laravel y una base de datos PostgreSQL.

\section{TECNOLOGÍAS UTILIZADAS}

En este trabajo se utilizaron diferentes sistemas para levantar el sistema de seguimiento, predicciones y alertas bajo el estándar de GTFS realtime. Para obtener los datos de posición de los GPS se utilizó Traccar, para obtener los datos de predicciones se utilizó TransiTime, finalmente, para visualizar los paraderos con la información de predicciones y alertas se utilizó OneBusAway. A continuación se mencionan algunas características de estos sistemas:

Traccar [4] es un software libre de seguimiento GPS gratuito, que soporta más de 110 diferentes protocolos de comunicación de GPS, y ofrece una aplicación Android llamada "Tracker for Traccar" que es para el rastreo de dispositivo móvil con GPS. Traccar funciona con una gran cantidad de dispositivos de rastreo e incluso se integra con Open GTS [5].

TransiTime [6] es un sistema que calcula las predicciones de los taxibuses, mediante el GTFS estático y los datos del GPS. TransiTime es un proyecto de código abierto que fue creado para ayudar a las agencias de tránsito a generar información de arribo más precisa y confiable en tiempo real para los pasajeros, e incluso en un entorno complicado de grandes ciudades con más de mil vehículos.

OneBusAway (OBA) [7-8] es un sistema de código abierto para el despliegue de información de tránsito en tiempo real. OBA utiliza el estándar GTFS para la descripción básica del sistema de transporte y recibe la información en tiempo real en distintos formatos incluyendo GTFS realtime, SIRI y ACS Orbital Orbcad [9]. Las nuevas tecnologías de la información, como los teléfonos inteligentes y los dispositivos móviles han permitido que OneBusAway incorpore un mayor número de características proporcionando información más detallada sobre paradas de taxibuses con el objetivo de ayudar a los pasajeros a encontrar y verificar ubicaciones de paradas de taxibuses.

OBA puede trabajar con información estática (GTFS) pero sus características más interesantes se puede ver, si se utiliza además información en tiempo real (GTFS realtime). Por ejemplo, en la ciudad de New York que está utilizando este software, las consultas ascienden a 30.000 consultas por minuto [7].

El back-end del servidor OBA está escrito en Java y utiliza una variedad de librerías estándar de desarrollo y un framework de código abierto en su implementación. El sistema se compone de 
varios módulos de servicio, cada uno de los cuales proporcionan funcionalidades específicas, que se acoplan utilizando el Framework Spring inversion-ofcontrol. La persistencia de objetos Java es manejada por el framework Hibernate sobre en una base de datos relacional. El contenedor de servlets Tomcat combinado con el framework web MVC de Apache Struts hace la mayor parte del trabajo pesado para la publicación basada en web. Las aplicaciones AJAX del lado del cliente están escritas principalmente con Google Web Toolkit, que compila el código fuente de Java en un Javascript optimizado [10].

Según los resultados de una encuesta de evaluación de OBA, se manifiesta un aumento general en la satisfacción de los consultados con el transporte público: hacen más viajes de tránsito semanalmente, pasan menos tiempo esperando el transporte, tienen una mayor sensación de seguridad personal al usar el transporte público y, a menudo, caminan más lejos para utilizar este transporte. Estos resultados son positivos en términos de aumentar el uso del transporte público, para reducir la congestión del tráfico, reducir el impacto ambiental del transporte y fomentar el desarrollo de las comunidades. Incluso, beneficiar la salud del usuario al tener que caminar más para poder usar este transporte [11-12].

En este contexto, el estándar GTFS es fundamental para estos sistemas, cuya base se define como un formato estándar de datos para la planificación de transporte público e información geográfica asociada a ellos. Las empresas pueden desarrollar aplicaciones, que consuman estos datos del GTFS de manera interoperable [13].

GTFS se compone de una serie de archivos de textos, comprimidos en un archivo ZIP. Cada archivo modela un aspecto particular de la información de tránsito, por ejemplo paradas, rutas, viajes, horarios, y otros datos relacionados con el tránsito [13].

La otra parte de esta base de estos sistemas es el GTFS realtime que es una especificación (desarrollado por Google) que permite a las empresas de transporte público estructurar los datos de tránsito de tiempo real. El protocolo GTFS realtime es una extensión del protocolo GTFS, el cual incorpora información acerca de las interrupciones de los servicios, la ubicación de los vehículos y tiempo de llegada esperada, esta información corresponde a los tres tipos de datos del protocolo GTFS realtime: alerta - posición - actualización de viaje [14].

Esta información se suministra en archivos de alimentación (feed) que se muestran a través de HTTP y se actualizan con frecuencia. Estos archivos en sí, son archivos binarios; por lo que, cualquier tipo de servidor web puede alojar y mostrar (es posible utilizar otros protocolos de transferencia también). Alternativamente, en posible utilizar servidores de aplicaciones que entregarían los archivos de alimentación (feed) como una respuesta a una solicitud GET de HTTP. No hay limitaciones en cuanto a la frecuencia ni al método exacto de cómo los archivos alimentación deben ser actualizados o recuperados, pero tienen que estar lo suficientemente actualizados para que el GTFS realtime represente el estado real de los vehículos [15].

El formato de intercambio de datos del GTFS realtime está basado en Protocol Buffer, el cual es un mecanismo de lenguaje y plataforma neutral para serializar datos estructurados (como XML, pero más pequeño, rápido y simple). La estructura de estos datos se define en un archivo gtfs-realtime.proto, que luego se utiliza para generar el código fuente, para leer y escribir fácilmente los datos estructurados, desde y hacia una variedad de flujos de datos. Cada mensaje basado en Protocol Buffer es un pequeño registro lógico de información, que contiene una serie de pares nombre-valor [16].

Protocol Buffers es un lenguaje para serializar datos estructurados, el cual ha mostrado ser mucho más eficiente que XML [16]. Todos los archivos de alimentación (feeds) de GTFS realtime (position, trip update y alerts) están en este lenguaje. La lectura de estos datos, que suelen venir en grandes volúmenes, requiere que se realice en el menor tiempo posible. Por esto, es relevante el uso de este lenguaje, que hasta el momento ha demostrado ser eficiente en la implementación de este tipo de soluciones.

En la Figura 1 se muestra un ejemplo de los archivos de alimentación (feed) del proyecto basado en este aporte [17].

\section{DESARROLLO}

La Figura 2 muestra los tres componentes básicos del sistema implementado: (1) el sistema de 

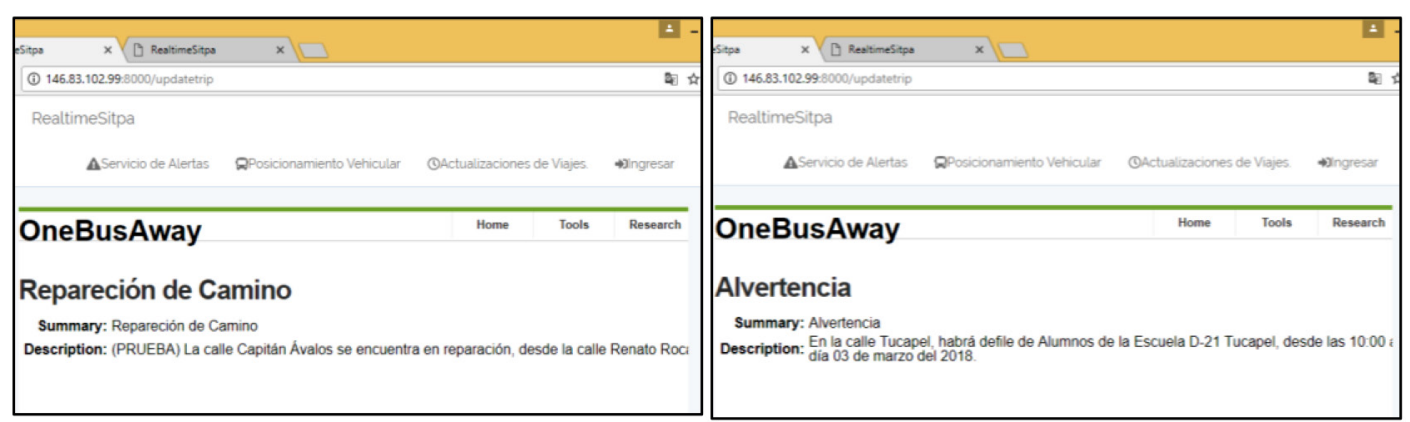

Figura 1. Feed del GTFS realtime.

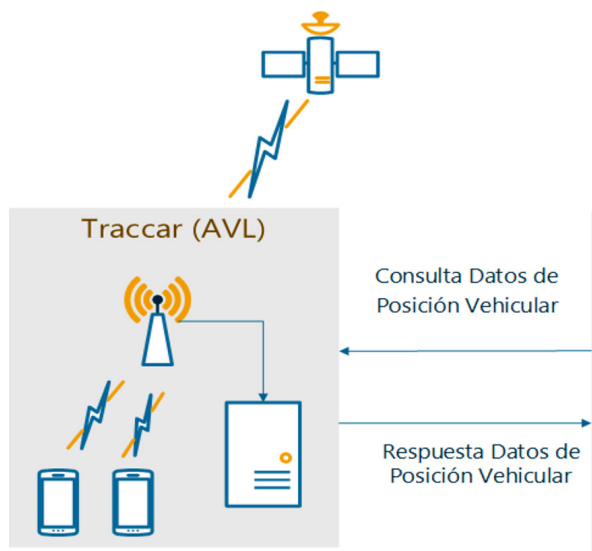

Figura 2. Arquitectura del Sistema.

localización vehicular automática (AVL); (2) el servidor GTFS realtime; (3) el sistema Web, el cual muestra las alertas, posiciones y actualizaciones de viaje. En el componente AVL se muestra un satélite, el cual, se comunica con los GPS de los celulares para determinar la ubicación a través de la triangulación y luego se envía a través de internet al servidor AVL. El servidor GTFS realtime consulta por los datos del GPS: latitud, longitud, velocidad y dirección, y el sistema AVL responde a esta consulta, luego el servidor GTFS Realtime prepara los datos según la especificación del GTFS realtime, en el lenguaje de Protocol Buffers [16], para que luego sean consultado por HTTP, a través de los sistemas Transitime y OneBusAway, los cuales, proveerán interfaces de seguimiento; alertas y actualizaciones de viaje en los paraderos.

Una descripción más en detalle se muestra en la Figura 3, la cual muestra los componentes y las relaciones de dependencia entre estos. La base de datos comparte dos esquemas: uno, para el sistema de AVL; y otro para el sistema en general. El sistema AVL tiene un servidor Tracker-server. jar y un aplicativo Tracker-traccar.apk. El servidor aplicación (servidor GTFS realtime) incluye un servidor apache2 Laravel y un conjunto de APIs, programas y archivo, para generar los datos de tránsito en tiempo real según las especificación de GTFS realtime. La capa de presentación representa las interfaces que se muestran en este sistema, como los servicios de alertas, seguimientos y actualizaciones de viajes.

En el servidor aplicación hay dos API, que permiten obtener información de la base de datos, que luego será transformada por el programa Generador GTFS realtime en archivos de alimentación para el GTFS realtime: alerta y posición. Los programas onebusaway.war y transitime.jar proveen, respectivamente, de los servicios de seguimiento y, alertas y actualizaciones de viajes. El archivo 


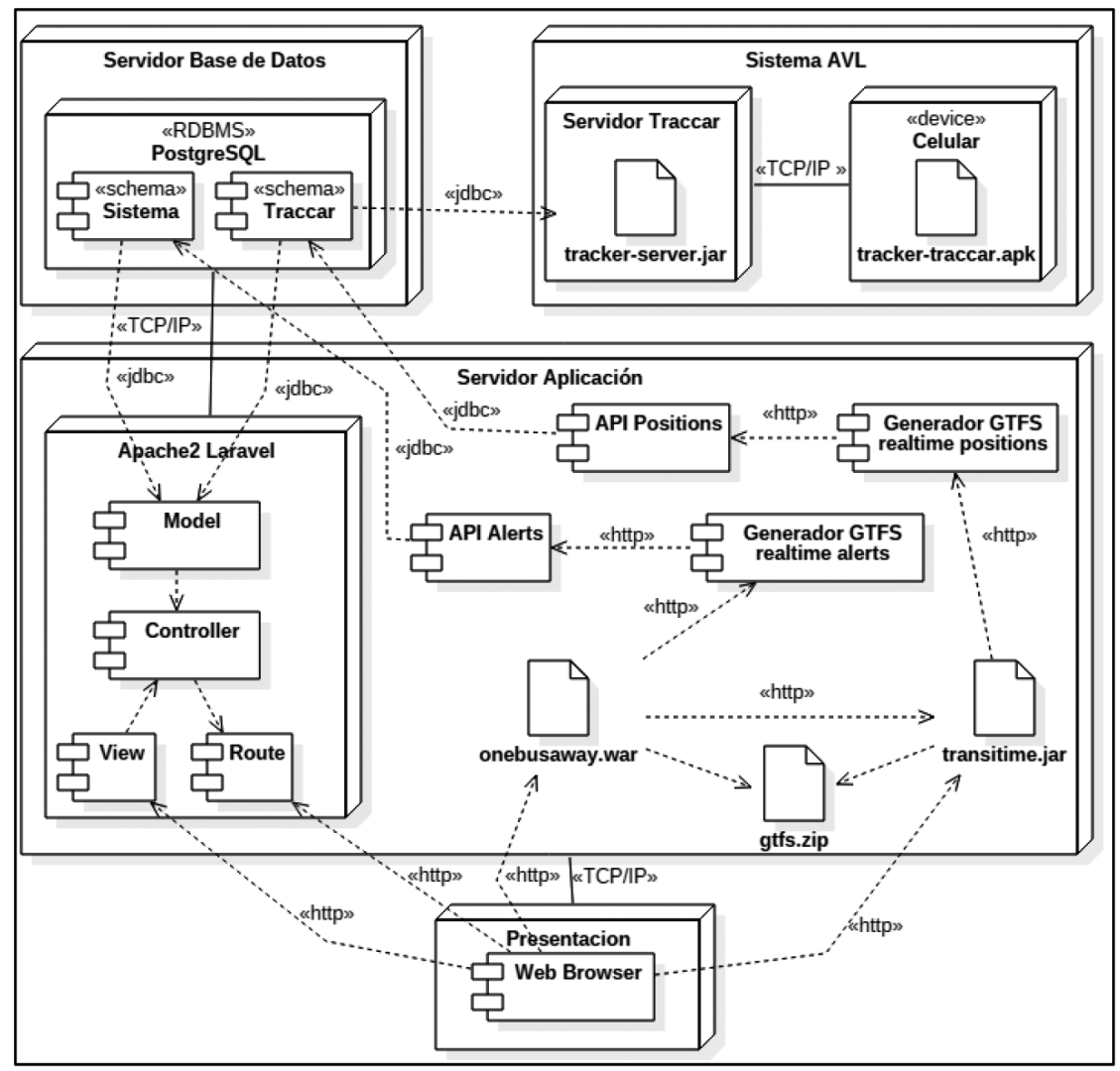

Figura 3. Diagrama de Despliegue.

gtfs.zip es utilizado por estos dos programas. El servidor Apache2 Laravel tiene la aplicación web para administrar el sistema en general.

En la Figura 4 se muestra las relaciones de estos componentes mediante interfaces, los cuales explican mejor el formato de los datos que se comparten. Las API muestran las interfaces en datos JSON para que el siguiente programa Generador GTFS realtime transforme estos datos en GTFS realtime (GTFS-rt). El programa transitime.jar recibe los datos de posición en formato según la especificación del GTFS realtime para otorgar el servicio de seguimiento en tiempo real y además producir datos de actualización de viaje en el lenguaje Protocol Buffers según la especificación GTFS realtime. El programa OneBusAway recibe los datos de alertas, posición y actualización de viaje; para proveer el servicio de interfaces de paraderos con información del alerta y actualización de viaje. Estos programas dependen del archivo GTFS.zip en el que se encuentra toda la información estática de tránsito.
La gestión de este sistema se realiza en el componente View, el cual, permite registrar nuevos usuarios con privilegio de ingresar las alertas asociadas a una ruta del sistema de tránsito y asociar a los GPS la ruta que está siguiendo. Además este componente llama vía HTTP a las interfaces provistas por los programas transitime.jar y onebusaway.war.

La Figura 5 describe las actividades asociadas al funcionamiento del sistema. La actividad Gestionar Alertas permite al usuario administrador ingresar las alertas al sistema y elegir las rutas que serán afectados por estas. La actividad Obtener Datos en JSON es una API que se dedica a obtener un abstracto de los datos de alertas de la Base de Datos en un Array de tipo JSON. La actividad Creando Feed Alertas en GTFS RT utiliza la API recién mencionada para transformar los datos de alertas en formato GTFS realtime. Por otro lado, la actividad Gestionar GPS permite, al usuario administrador, actualizar las rutas asociadas a los GPS para que luego puedan ser transformado en formato GTFS realtime. 


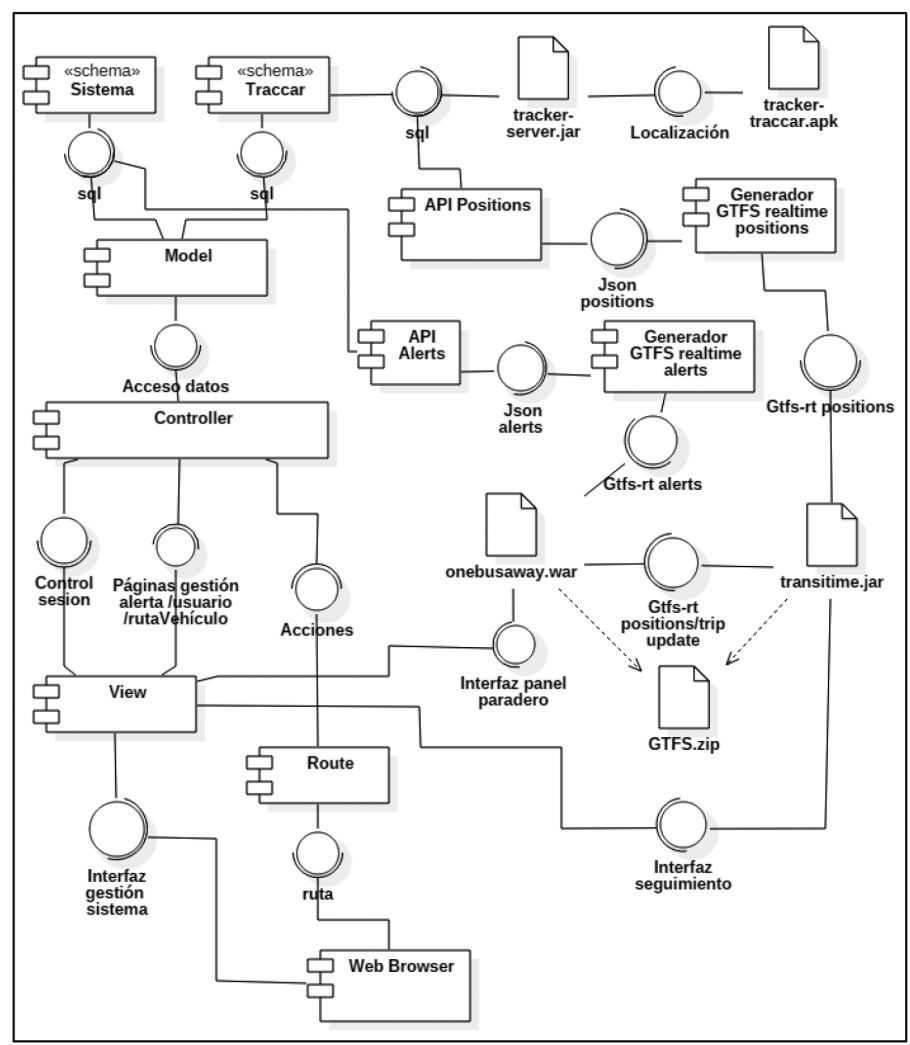

Figura 4. Diagrama de Componentes.

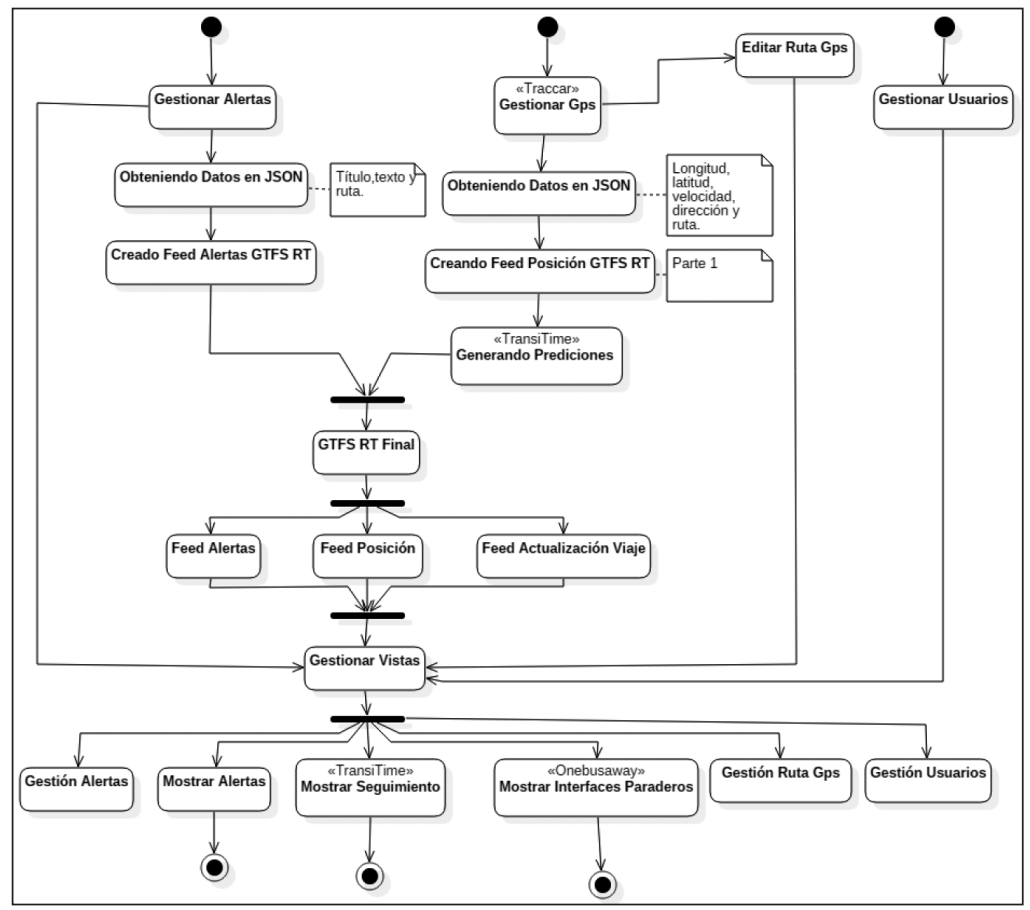

Figura 5. Diagrama de Actividades. 
A partir de estos datos, la actividad Generando Predicciones (transitime.jar) produce la información de actualización de viajes. Finalmente, a partir de la información producida anteriormente, el programa transitime.jar podrá mostrar el seguimiento de los Taxibuses y el programa onebusaway.war podrá mostrar los tiempos de llegada a los paraderos como sus alertas asociadas.

\section{ANÁLISIS DE RESULTADOS}

En esta sección se muestran tres pruebas realizadas para probar la correctitud de la información entregada por el sistema: alerta, posición y actualización de viaje. La primera prueba se diseñó para probar el correcto funcionamiento del sistema con dos GPS (taxibuses) recorriendo diferentes rutas simultáneamente. La segunda prueba se diseñó para probar el correcto funcionamiento del sistema con dos GPS (taxibuses) recorriendo la misma ruta. La tercera prueba se realizó comparando el comportamiento del sistema desarrollado con la información entregada por Sistema de Transito de Google Maps de la ciudad de Arica, el cual utiliza información del GTFS realizado por el proyecto SITPA [1] de la Universidad de Tarapacá.

Estas pruebas mostraron el adecuado seguimiento de los taxibuses y, adicionalmente, que las estimaciones de llegadas a los paraderos están acordes a la realidad. Así como también, que las alertas se encuentran correctamente asociadas a la ruta afectada.

\section{Experimento 1}

En la Figura 6 se observa el movimiento de los taxibuses (líneas 4 y 10) y su dirección de desplazamiento, mostrando el correcto funcionamiento del seguimiento a los taxibuses considerando el uso de los datos de GTFS realtime posición.

En la Figura 7 se observa la información de los dos taxibuses en movimiento. La figura de la izquierda corresponde al taxibus de la línea 4 y la figura de la derecha al taxibus de la línea 10.

También se probaron los datos de tiempo de llegada en las interfaces correspondientes a los paraderos. En la Figura 8 se observa la información asociada a los paraderos "Argentina / La Exótica" y "Lindero / Villarrica", asociados, respectivamente, a las líneas 4 y 10.

En la Figura 9 se observa la información de predicción de estos paraderos. Los minutos que se observan en gris son estimaciones asociados a datos GTFS y los minutos que se ven en color azul son estimaciones realizadas a partir de datos GTFS realtime. En esta figura se observan las alertas que han sido ingresadas considerando el uso de los datos GTFS realtime y corresponden a la ruta afectada.

Accediendo mediante el icono de alertas se observa el detalle de cada una de estas (ver la Figura 10). Esto permite que el usuario pueda tener información en tiempo real de las alertas que han sido ingresadas por el usuario administrador.

\section{Experimento 2}

En la Figura 11 se observa el movimiento de dos taxibuses y su dirección de desplazamiento siguiendo

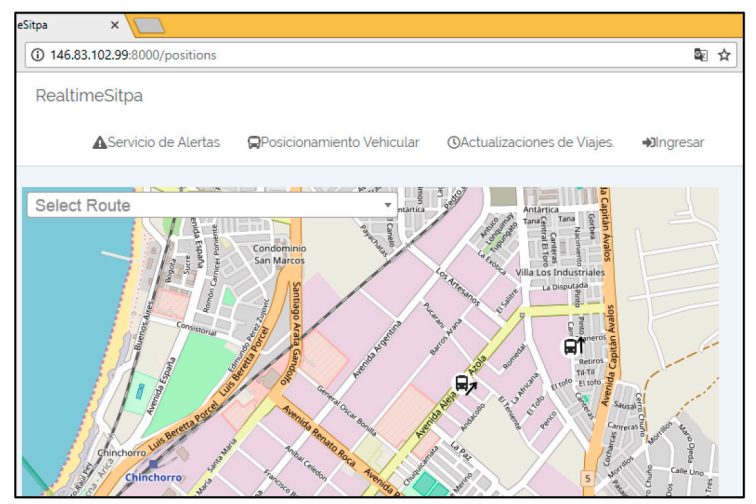

Figura 6. Experimento 1, Seguimiento de los Taxibuses L4 y L10. 


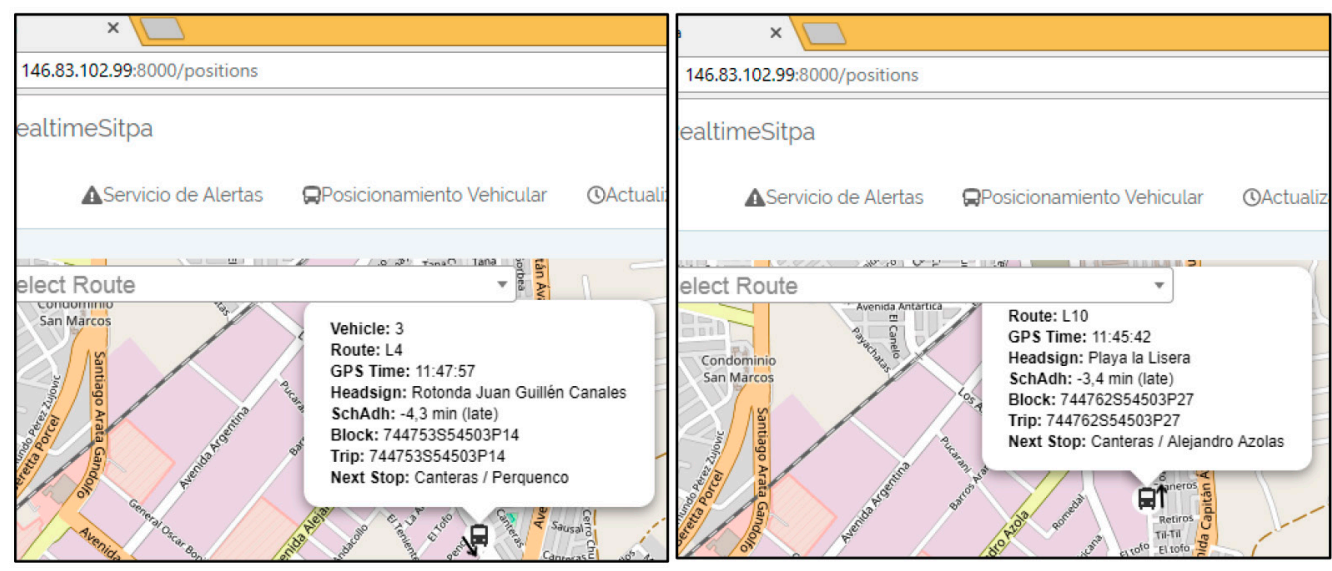

Figura 7. Experimento 1, Información de los Taxibuses L4 y L10.

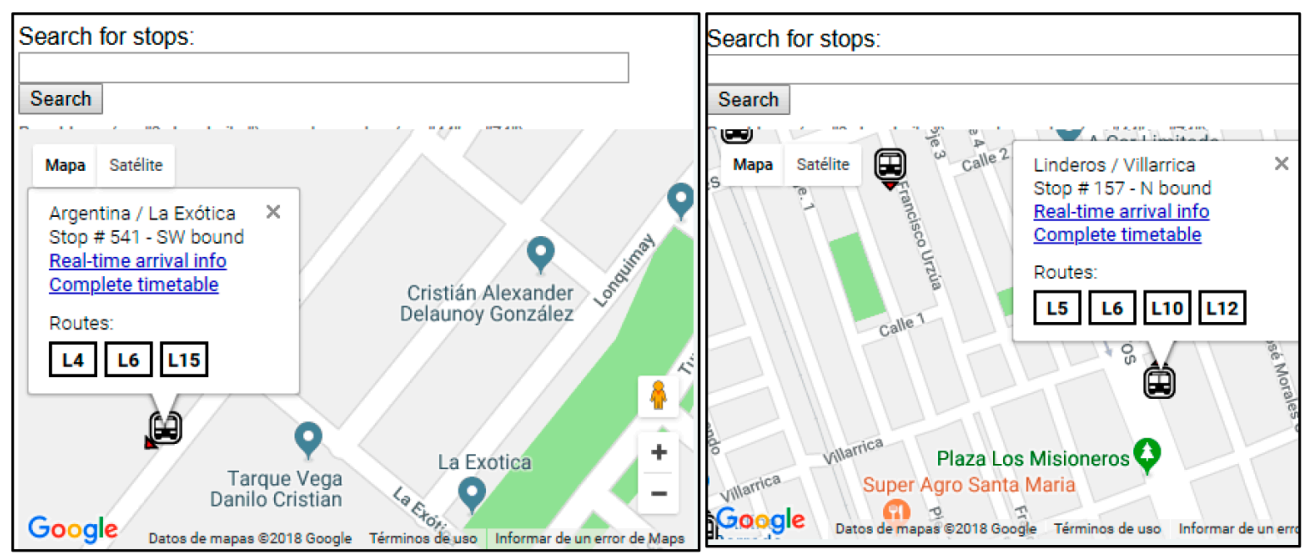

Figura 8. Experimento 1, Observación de Paraderos.

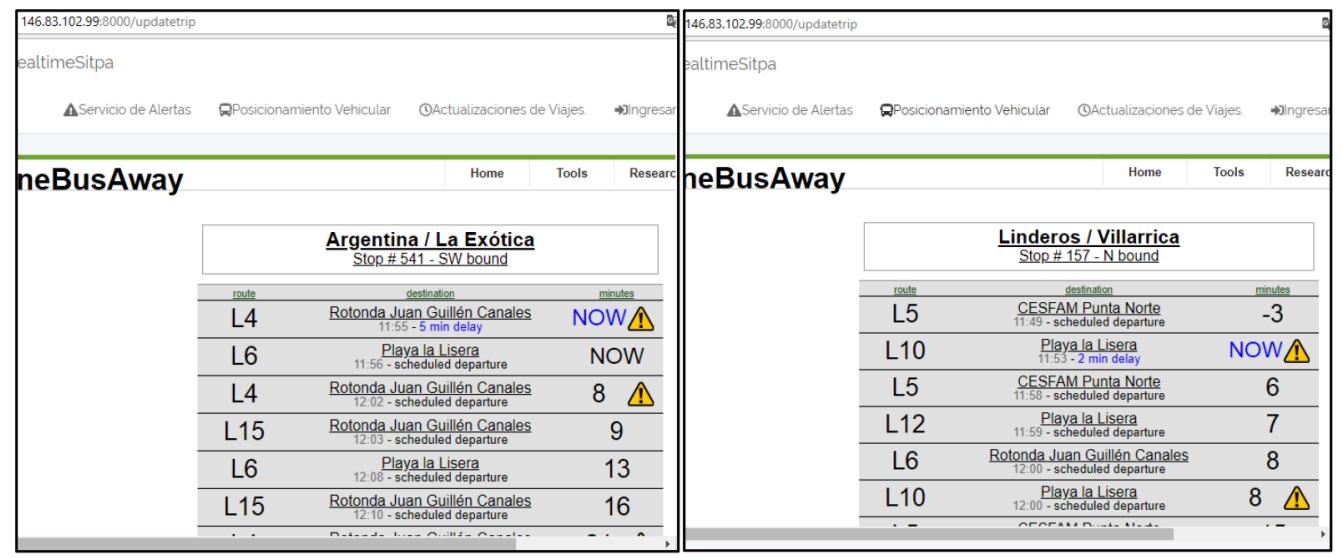

Figura 9. Experimento 1, Observación del Tiempo de Llegada. 

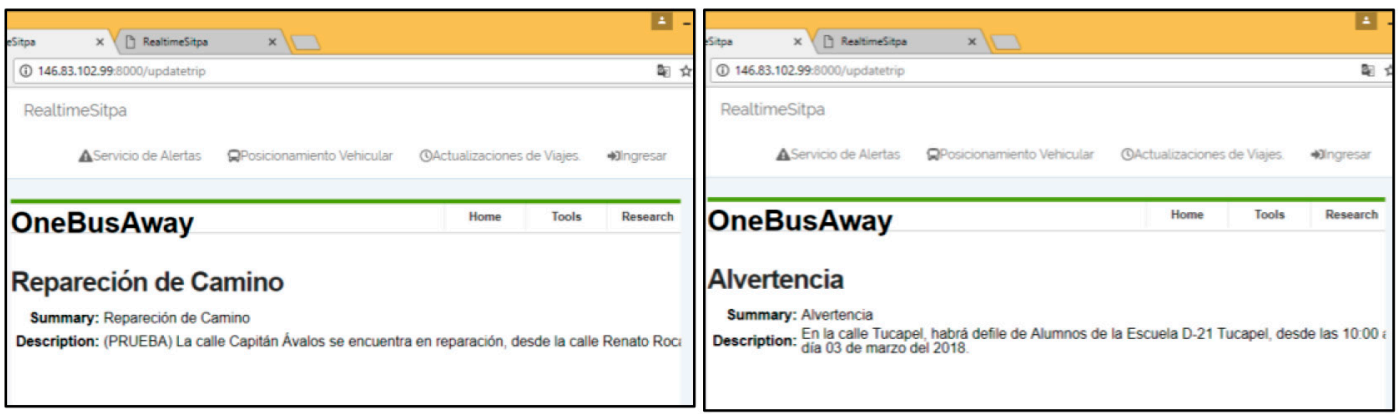

Figura 10. Experimento 1, Alertas en el Sistema de Onebusaway.

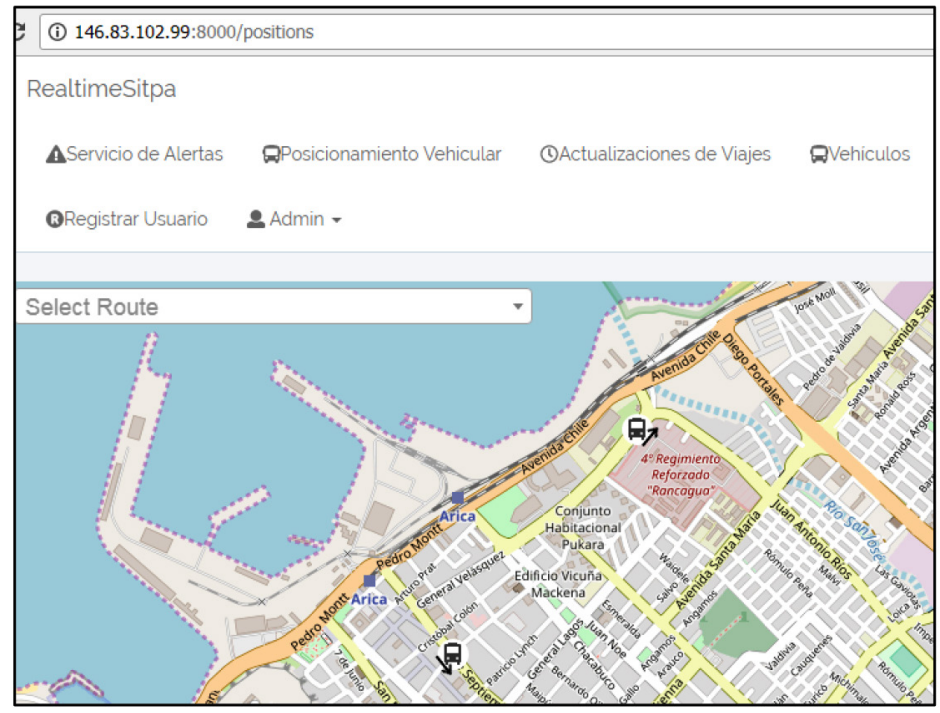

Figura 11. Experimento 2, Seguimiento de los Taxibuses L12.

la ruta de la línea 12 mediante el uso de los datos de GTFS realtime posición.

En la Figura 12 se muestra la información de los dos taxibuses en movimiento. La figura de la izquierda corresponde al primer taxibus y la figura de la derecha al segundo taxibus, ambos de la línea 12.

En la Figura 13 se muestra el paradero "Luis Beretta / Brasil", por el cual, pasan ambos taxibuses de la línea observada.

En la Figura 14 se observan la información de predicciones de este paradero. Dado que, solamente se han usado dos GPS, solo es posible observar dos estimaciones en tiempo real, el resto corresponde a estimaciones del GTFS estático. La primera columna muestra las líneas de los taxibuses que están por pasar, la segunda columna las horas en que estos irán a pasar como también los minutos de atraso o adelanto del taxibus, y la tercera columna el tiempo que el taxibus tardará en pasar por este paradero. En esta figura se observa que el primer taxibus está pasando en ese mismo momento (NOW), mientras que el segundo Taxibus pasará en 8 minutos más.

\section{Experimento 3}

En la Figura 15 se puede observar los tiempos de llegada de los taxibuses al paradero "Luis Beretta/ Brasil" utilizando del Sistema de Transito Google Maps de la ciudad de Arica. Se observa que los tiempos de llegada para la línea 12 son: 13:37, 13:47 y $13: 58$. 


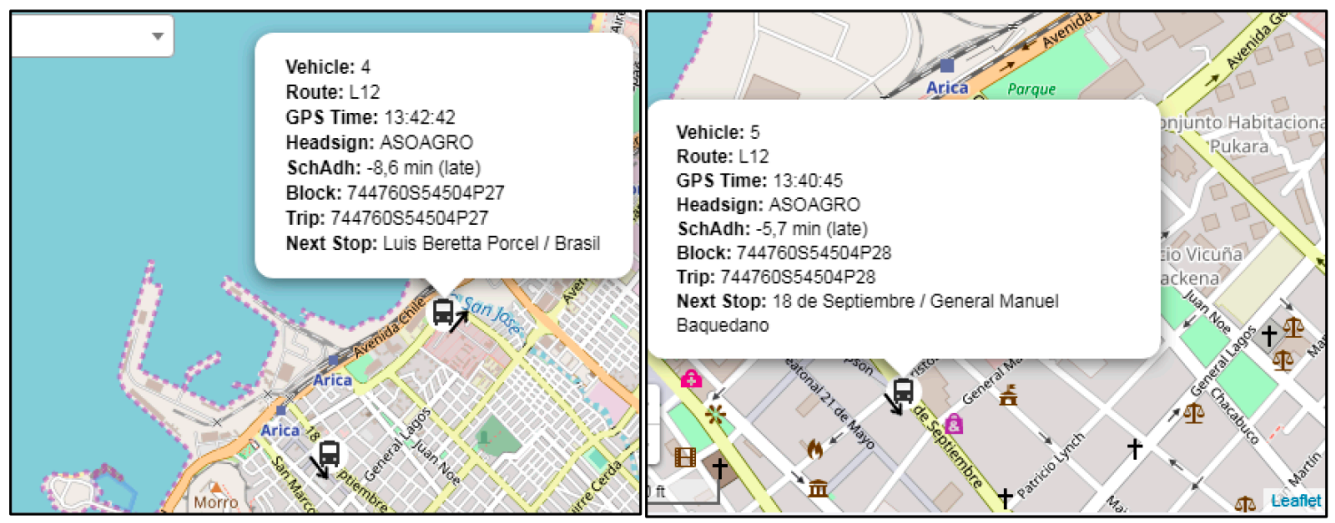

Figura 12. Experimento 2, Información de los Taxibuses L12.

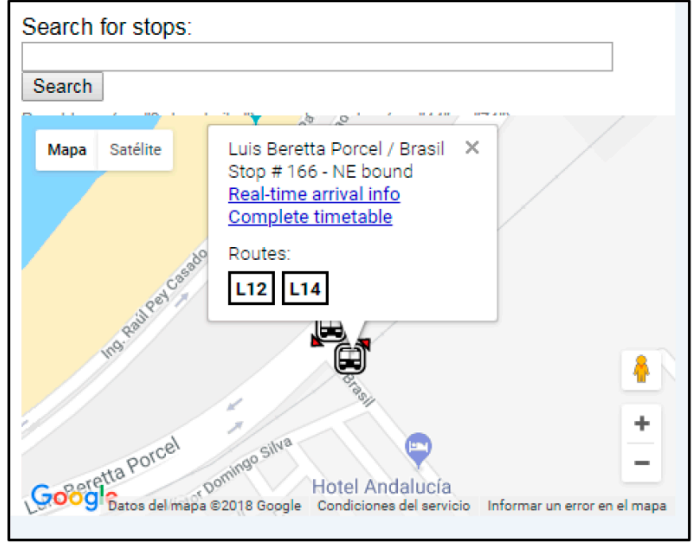

Figura 13. Experimento 2, Observación de Paradero.

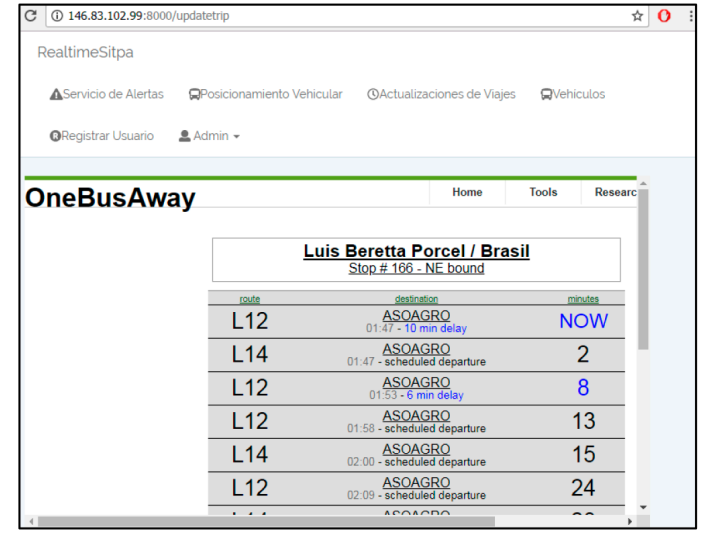

Figura 14. Experimento 2, Observación del Tiempo de Llegada.

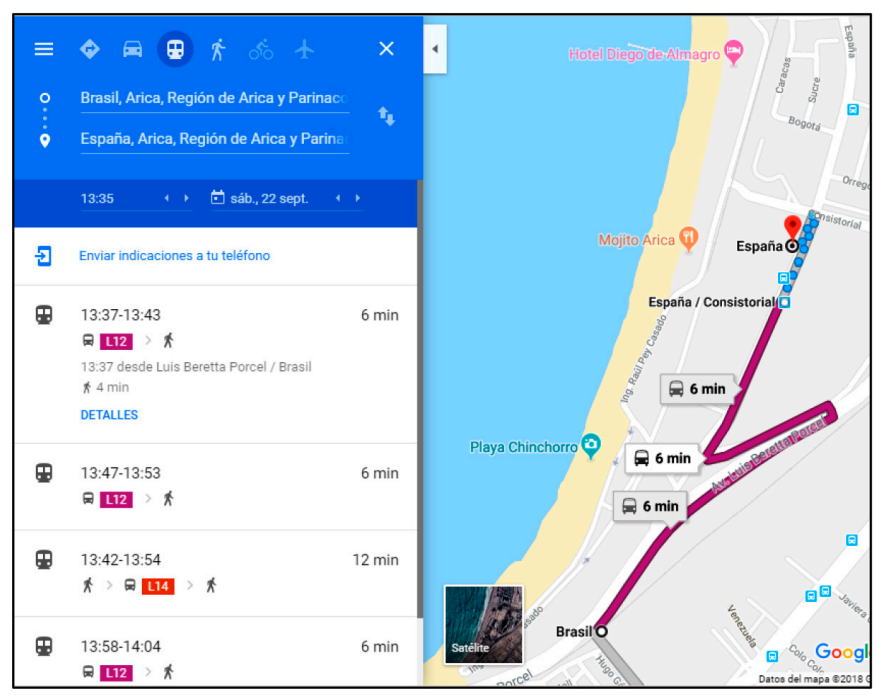

Figura 15. Tiempo de la Parada. 
Comparando esta información con la mostrada en la Figura 14, para el mismo paradero "Luis Beretta/ Brasil", se observa que los tiempos de llegada para los taxibuses con GPS son: 13:47 y 13:53, tiempos que corresponden a predicciones de llegada. La Tabla 1 muestra una comparativa para estos taxibuses considerando los casos GTFS (Google Maps) y GTFS realtime.

Tabla 1. Comparativa GTFS vs GTFS realtime.

\begin{tabular}{|l|c|c|c|}
\hline & GTFS & \multicolumn{2}{|c|}{ GTFS realtime } \\
\hline & $\begin{array}{c}\text { Tiempo } \\
\text { Llegada }\end{array}$ & $\begin{array}{c}\text { Tiempo } \\
\text { Llegada }\end{array}$ & $\begin{array}{c}\text { Tiempo de } \\
\text { Atraso }\end{array}$ \\
\hline Taxibus Uno & $13: 37$ & $13: 47$ & $10 \mathrm{~min}$. \\
\hline Taxibus Dos & $13: 47$ & $01: 53$ & 06 min. \\
\hline
\end{tabular}

El tiempo de llegada del primer taxibus estaba programado a las 13:37 según el GTFS, pero este se encuentra atrasado en 10 minutos, por lo tanto, pasará a las 13:47, como lo dice el GTFS realtime. Lo mismo sucede con el siguiente taxibus, que tiene un atraso de 6 minutos.

Las predicciones del GTFS realtime corresponden a una estimación de tiempo más precisa con respecto a la llegada de un taxibus a un paradero, por lo que disponer de esta información es relevante para los usuarios del transporte público, los que pueden hacer una mejor gestión de su tiempo.

\section{CONCLUSIONES}

El concepto de Ciudades Inteligentes ha ido tomando importancia y gran relevancia a la hora de trazar e implementar políticas públicas a nivel mundial dado el desarrollo y crecimiento poblacional de los centros urbanos [18] es por tal motivo que se implementó un sistema SIG-T que constituye una herramienta fundamental para ordenar, clasificar y planificar distinto tipos de datos sobre las ciudades [19].

El Sistema de Información desarrollado incorpora el uso del estándar GTFS realtime e integra herramientas de software de libre disposición (TransiTime, OneBusAway y Traccar), con lo que se espera aportar a la operación del servicio de trasporte público de la ciudad de Arica, el que se caracteriza por la variabilidad que presenta tanto en los tiempos de viaje entre paraderos sucesivos como en la demanda de pasajeros en cada uno de ellos [20].

Es importante mencionar que, para el correcto funcionamiento de este tipo de sistemas, la información capturada debe proveerse en Protocol Buffers, cumpliendo con la especificaciones del GTFS realtime. Con este tipo de información, el sistema descrito es capaz de entregar como resultado el seguimiento de los taxibuses, las actualizaciones del tiempo de arribo a cada paradero y un servicio de alerta tomando en consideración la variabilidad que se pueda presentar en los tiempos de viaje.

Existen diferentes tipos de datos para el intercambio de información en tiempo real. En este trabajo se optó por el uso de GTFS realtime dado que se tenía como experiencia previa el desarrollo de SITPA [1], un sistema SIG de planificación de viajes, basado en el uso de un GTFS estático. Adicionalmente, es la especificación oficial utilizada en la plataforma Google Maps.

Cabe recalcar que actualmente variadas herramientas usadas en el desarrollo de soluciones SIG-T utilizan el estándar GTFS realtime, por ejemplo: OpenTripPlanner, TransiTime y OneBusAway.

A futuro se espera integrar a SITPA los datos provistos por este sistema, para que la ciudad de Arica tenga una planificador de viaje en tiempo real. Además, es interesante considerar la incorporación de un sistema que pueda proveer datos del nivel de congestión vehicular en diferentes calle de la ciudad, cuyos datos puedan ser agregado al GTFS realtime desarrollado en esta investigación. Finalmente, el uso de este estándar posibilita ofrecer la información que genera el sistema a la empresa Google para que pueda entregar las planificaciones de viajes con estimaciones en tiempo real a través de su aplicación Google Maps.

\section{REFERENCIAS}

[1] M. Navarrete, R. Valdivia y R. Cornejo. "Sistema de información para la Gestión del Transporte con Tecnologías de Libre Disposición". XV Conferencia Iberoamericana de Sistemas de Información Geográfica. Vol. 1, pp. 295-305. 9, 10 y 11 Septiembre 2015. 
[2] R. Valdivia y M. Navarrete. "Estándares abiertos e interoperabilidad: el caso GTFS". Ingeniare. Revista chilena de ingeniería. Vol. $24 \mathrm{~N}^{\circ} 2$, pp. 186-187. Abril, 2016. DOI: 10.4067/S0718-33052016000200001.

[3] J.-C. Thill. "Geographic Information Systems in Transportation Research". Oxford: Pergamon. Vol. 8, pp. 3-12. 2000.

[4] TRACCAR. "TRACCAR". 18 de septiembre 2018. URL: https://www.traccar.org/

[5] G. Hoppe. "The 6 Best Free and Open Source Fleet Management Software Programs". Capterra. 18 de septiembre 2018. URL: https:// blog.capterra.com/the-6-best-free-and-opensource-fleet-management-software-programs/

[6] M. Smith. "GitHub - TransiTime". Fecha de consulta: 18 de septiembre 2018. URL: https://github.com/Transitime

[7] OneBusAway. "OneBusAway". Fecha de consulta: 18 de septiembre 2018. URL: http://onebusaway.org/

[8] M. Campbell, C. Bennett, C. Bonnar and A. Borning. "Where's My Bus Stop? Supporting Independence of Blind Transit Riders with StopInfo". ACM ASSETS Conference, ACM, 2014.

[9] TransitWiki. "OneBusAway". Fecha de consulta: 18 de septiembre 2018. URL: https://www.transitwiki.org/TransitWiki/ index.php/OneBusAway

[10] B. Ferris, K. Watkins and A. Borning. "OneBusAway: A Transit Traveler Information System". In: T. Phan, R. Montanari, P. Zerfos. (eds). Mobile Computing, Applications, and Services. MobiCASE 2009. Lecture Notes of the Institute for Computer Sciences, Social Informatics and Telecommunications Engineering. Vol. 35. 2010.

[11] B. Ferris, K. Watkins and A. Borning. "OneBusAway: Results from Providing Real-Time Arrival Information for Public
Transit". ACM Conference on Human Factors in Computing Systems (CHI). New York, USA. 2010.

[12] J.-M. Seguí Pons y M.R. Martínez Reynés. "Los sistemas inteligentes de transporte y sus efectos en la movilidad urbana e interurbana". Geo Crística / Scripta Nova. Revista electrónica de geografía y ciencias sociales. Vol. 8, pp. 170-60. 01 Agosto 2004.

[13] Google Transit. "GTFS Static Overview". Fecha de consulta: 18 de septiembre 2018. URL: https: //developers.google.com/transit/gtfs

[14] TransitWiki. "Transit Wiki GTFS realtime". Fecha de consulta: 18 de septiembre 2018. URL: https://www.transitwiki.org/ TransitWiki/index.php/GTFS-realtime

[15] Google Transit. "GTFS REAL TIME". Fecha de consulta: 18 de septiembre 2018. URL: https://developers.google.com/transit/ gtfs-realtime/

[16] Google. "Protocol Buffers". Fecha de consulta: 18 de septiembre 2018. URL: https:// developers.google.com/protocol-buffers/

[17] F.M. Alves. "Relatório Final de Estágio Obrigatório". Universidade Federal do Pampa, Bagé - Brazil. 2016.

[18] G. Maestre. "Revisión de literatura sobre ciudades inteligentes: una perspectiva centrada en las TIC". Ingeniare. Vol. 11 Issue 19, pp. 137-149. 2015.

[19] E. Bustillo Holgado y P. Rodríguez Bustamante. "Los Sistemas de Información Geográfica y las ciudades inteligentes". Polígonos. Revista de Geografía. No 27 , pp. 257-270. 2015.

[20] F.A. Zúñiga González. "Estimación y Predicción de Matrices dinámicas de viaje sobre un corredor de Transporte Público, utilizando Datos Históricos e Información en Tiempo Real". Tesis Magíster. Santiago, Chile. 2011. 